\title{
Nursing schools and academic health centers: toward improved alignment and a synergistic partnership
}

This article was published in the following Dove Press journal:

Journal of Multidisciplinary Healthcare

7 August 2017

Number of times this article has been viewed

\author{
Azita Emami' \\ Darcy Jaffe ${ }^{2}$ \\ Paula Minton-Foltz ${ }^{3}$ \\ Grace Parker ${ }^{4}$ \\ Susan Manfredi ${ }^{5}$ \\ Theresa Braungardt ${ }^{6}$ \\ Kelly W Marley' \\ Laura Cooley' \\ Staishy Bostick Siem ${ }^{7}$ \\ 'University of Washington School \\ of Nursing, Seattle, WA, USA; \\ ${ }^{2}$ Harborview Medical Center, \\ University of Washington Medicine, \\ Seattle, WA, USA; ${ }^{3}$ Patient Care \\ Services, Harborview Medical Center, \\ University of Washington Medicine, \\ Seattle, WA, USA; ${ }^{4}$ University of \\ Washington Medical Center, Seattle, \\ WA, USA; ${ }^{\text {PPatient }}$ Care Services, \\ Northwest Hospital and Medical \\ Center, Seattle, WA, USA; ${ }^{\circ}$ Valley \\ Medical Center, Seattle, WA, USA; \\ ${ }^{7}$ Marketing and Communications, \\ University of Washington School of \\ Nursing, Seattle, WA, USA
}

Correspondence: Azita Emami University of Washington School of Nursing, Health Sciences, Box 357260, 1959 NE Pacific St, T-30I, Seattle, WA 98195-7260, USA

$\mathrm{Tel}+|20622| 2472$

Email Sondean@uw.edu
Abstract: This paper presents the findings from a national survey which the University of Washington conducted among leaders of 32 US academic nursing institutions that are part of academic health centers (AHCs) and complements these findings with results from a separate report by the American Association of Colleges of Nursing. While expressing overall satisfaction with their AHC relationships, these leaders find that nursing is often given greater parity in matters of education and research than in mission setting, financial, and governance matters. AHCs are being asked to meet new health care challenges in new ways, starting with the education of health care professionals. AHCs need to be restructured to give nursing full parity if the nation's and world's needs for preventive and clinical care are to be best met.

Keywords: nursing parity, academic nursing institutions, nurse leaders, institutional alignment

\section{Background}

Academic health centers (AHCs) are defined by the Association of Academic Health Centers (AAHC) as consisting of an allopathic or osteopathic medical school, at least one other health professions school or program, and at least one affiliated or owned teaching hospital. ${ }^{1}$ In most cases, an academic nursing institution (ANI) is one of the health professional programs of an AHC, contributing to all aspects of the AHC's integrated triad of education, research, and clinical endeavors.

The relationship between AHCs and ANIs is becoming increasingly important as the role of AHCs and nursing changes to meet contemporary health care needs, not only in the US but also globally. A report by the Institute of Medicine's Committee on the roles of AHCs in the 21 st century observed that, "While academic health centers (AHCs) have made important contributions to the health of people in this nation and internationally, there is no question that the future will present a very different set of demands on these institutions." 2

Nurses comprise $60 \%-80 \%$ of the total health system worldwide and provide $90 \%$ of all health care services. ${ }^{3}$ ANIs are a key element in interprofessional education programs that are now a prominent part of health care education at virtually every institution. However, the potential for full engagement of schools of nursing within academic health systems or centers has often been hampered by structural, managerial, and sociocultural barriers. The evolving role of nurses worldwide, including their increasing role as primary care providers, makes it important that we have a better understanding of the relationship between schools of nursing and the AHCs of which they are often a part. 


\section{Methods}

In 2015, the University of Washington School of Nursing (UWSoN) commissioned the "Nursing Schools and Academic Health Center Relationship Survey", a national inquiry to gather perspectives about AHCs from nursing school leaders situated within US AHCs. The primary purpose of the survey was to understand the roles that nursing schools play in the planning, leadership, and operational functions of AHCs.

The survey consisted of 20 questions. Participants were deans or their designees, representing public and private ANIs throughout the US. Participants were assured of confidentiality and anonymity. Under University of Washington Institutional Review Board (IRB) rules, the survey did not require IRB review or approval.

The survey was conducted on behalf of the UWSoN by a private firm (Hanover Research). It was offered online to a convenience sample of nursing administrators at 76 ANIs associated with an AHC that was a member of the AAHC. The response rate of $42 \%$ was robust for a small survey, with 32 full or partial surveys completed.

The survey was comprised of questions using Likertscale response options as well as open-ended questions to elicit qualitative data about the respondents' perceptions of relationship quality within their AHC and matters of governance and operations.

At about the same time, the American Association of Colleges of Nursing (AACN) hired a private firm, Manatt Health Solutions, to conduct a comprehensive, nationwide survey on the relationship between schools of nursing and AHCs with which their membership is affiliated. The AACN survey had an explicit aim of developing "an aspirational future vision for academic nursing in the context of evolving AHCs". ${ }^{4}$

The resulting report, referred to as the "Manatt Report", involved 48 interviews at 25 institutions. Interviews were conducted with 25 nursing school deans, five medical school deans, 16 health system Chief Nursing Officers and Chief Executive Officers, and eight university chancellors/vice chancellors. The final report was issued in 2016. It identified the areas where rebalancing was needed in order to address barriers to alignment, and offered other recommendations based on survey findings. ${ }^{5}$

\section{Results}

\section{Perceptions of relationship quality within AHCs}

The UWSoN survey responses revealed respondents perceive a generally positive - though sometimes ambivalent and tentative - relationship between schools of nursing and the AHCs with which they are affiliated. Perceptions were measured according to how respondents answered questions about their relationship quality with the $\mathrm{AHC}$ and the extent to which they felt involvement with an aspect of the AHC.

Forty-five percent of the respondents said that their ANI's relationship with the AHC was very positive, while an almost equal number (42\%) found it somewhat positive, for an overall positive rating of $87 \%$. However, when asked, "How satisfied is your nursing school with its overall level of involvement with the AHC?" nearly half (49\%) said they were either "very" or "somewhat dissatisfied". Only 39\% reported they were "somewhat" or "very satisfied" with their school's level of involvement.

By contrast, $48 \%$ of respondents felt that their school's relationship with the AHC was either "somewhat" or "much improved" over the last 5 years. Asked to cite positive aspects of their relationship with the AHC, $48 \%$ responded that they felt they were included in decision making; $41 \%$ stated that they support the AHC's mission; $37 \%$ cited as positive the communication/collaboration within AHCs; $26 \%$ found relationship building to be a positive; and $22 \%$ appreciated the recognition within AHCs that they received.

Qualitative responses related to relationship quality included:

Input in decisions, good communication, and relationships with other health science disciplines.

Participation in strategic planning and respect for the goals and mission of the school of nursing.

We have an active working relationship regarding nursing and nursing education. We interact across the system of the AHC. Our contributions are valued.

Qualitative responses about how relationships could be improved included:

AHCs are now threatened due to the changing payment structure. So asking for money is not going to strengthen the relationship. But having skills, knowledge, and competences that are of value within the system and offering collaborative partnerships which benefit both will strengthen the relationship. AHCs are also engaged with multiple schools of nursing, so we have to demonstrate our value as a preferred partner.

More involvement in leadership and more joint positions. There has been discussion to have faculty and students join nursing committees at $\mathrm{AHC}$ but that has not happened yet.

It should be a requirement for [the Joint Commission] and other accreditors that AHCs partner with colleges of nursing, public health, and medicine. 
Governance needs to change; there should also be shared visioning between two institutions.

\section{Perceptions of involvement in governance and operations}

To gauge perceptions of involvement in AHCs, respondents were asked, "What level of involvement does your nursing school have in the governance and operation of the AHC?" in each of four areas. The results (Figure 1) show that nurse leaders from schools of nursing perceive that they are more often invited to participate in matters of education and research (eg, as shown in the top two bar charts of Figure 1). However, in matters of decision making on strategic initiatives, $26 \%$ felt that they were "not at all involved" in key decision making. Nearly half - a total of $45 \%$ - reported they felt that they were "somewhat involved" or "not at all involved" in designing effective models for team-based collaborative care and optimizing critical outcomes.

When asked about the nursing school's role in governance and operation of the AHC as it relates to specific areas (Figure 2), 39\% reported they were "very involved" in research initiatives, and $32 \%$ indicated they were "very involved" in leadership. In contrast, lower levels of involvement of nursing schools were reported in other areas surveyed. For example, only about a quarter of the respondents felt "very involved" in mission setting (26\%) and development/fundraising (26\%), while for operations $(6 \%)$, and marketing $(6 \%)$ only a small percentage of the people felt very involved.

Despite respondents' sense of low levels of involvement in some finance-related matters of AHCs, when asked about the general areas of activity where they receive support from the $\mathrm{AHC}, 77 \%$ of the respondents cited joint research or clinical projects/programs; $57 \%$ named joint positions between school and $\mathrm{AHC}$; and 57\% noted they received financial contributions in exchange for work/research. Nevertheless, $10 \%$ of the respondents said that their ANI receives none of the specified support from its AHC.

When asked "How satisfied is your nursing school with various types of specific support?", only $20 \%$ indicated they were either "somewhat" or "very satisfied" with their joint research or clinical projects/programs. For joint positions between schools and AHCs, financial contributions in exchange for work/research, financial donations, and magnet status, the percentage of respondents who said that they were either "somewhat" or "very satisfied" ranged from $8 \%$ to $14 \%$.

A last area of inquiry in the UWSoN survey examined what would enhance the ANI/AHC relationship. One-third $(32 \%)$ of the respondents said more/better representation was the key; $63 \%$ of the respondents said their nursing school does not have a representative on the AHC's board of directors. Of the participants who answered they had board representation, only one held a position of board leadership, two were ex officio members, five were members at large, and one was on a work committee.

In response to open-ended questions related to what might strengthen the relationship with AHCs, respondents cited a need for increased collaboration, along with a desire for recognition of value, an equal partnership, and a need to formalize the relationship. Respondents expressed interest in increasing joint appointments and improving communication across AHCs.

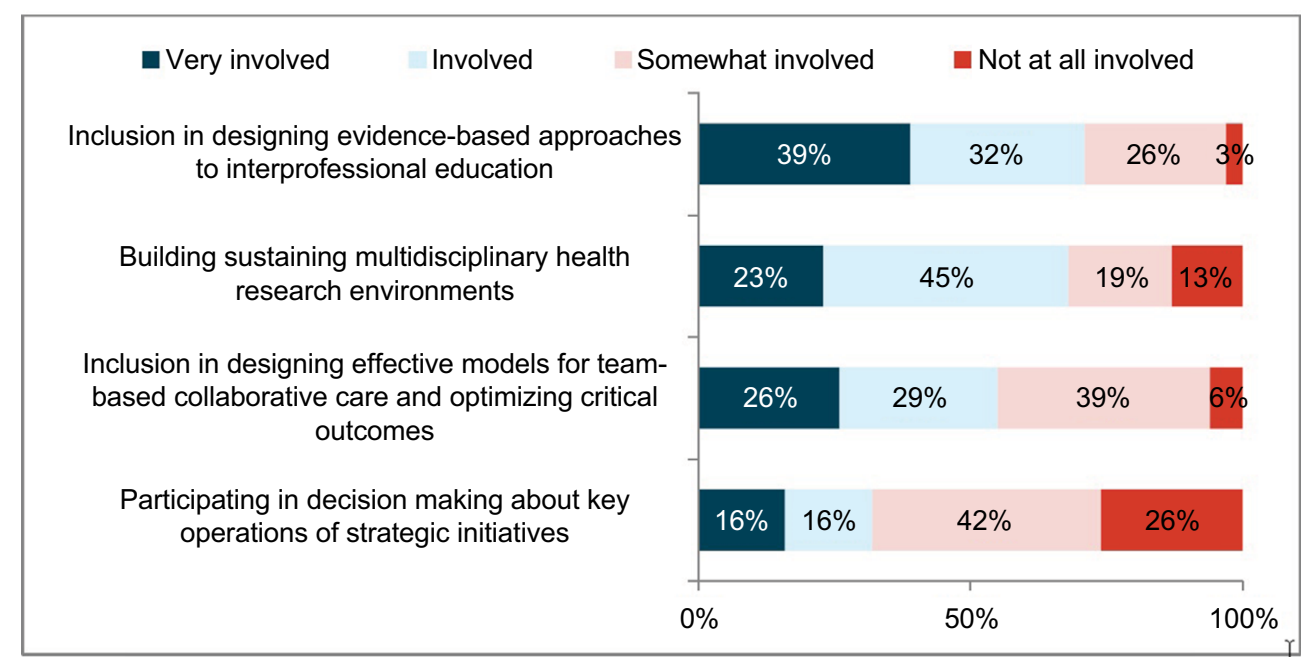

Figure I Perceived levels of involvement of schools of nursing in AHCs in the US.

Note: This chart is adapted from the report of Hanover Research, "Nursing School and AHC Relationship Survey Analysis," commissioned by the University of Washington School of Nursing, March 2015.

Abbreviation: AHCs, academic health centers. 


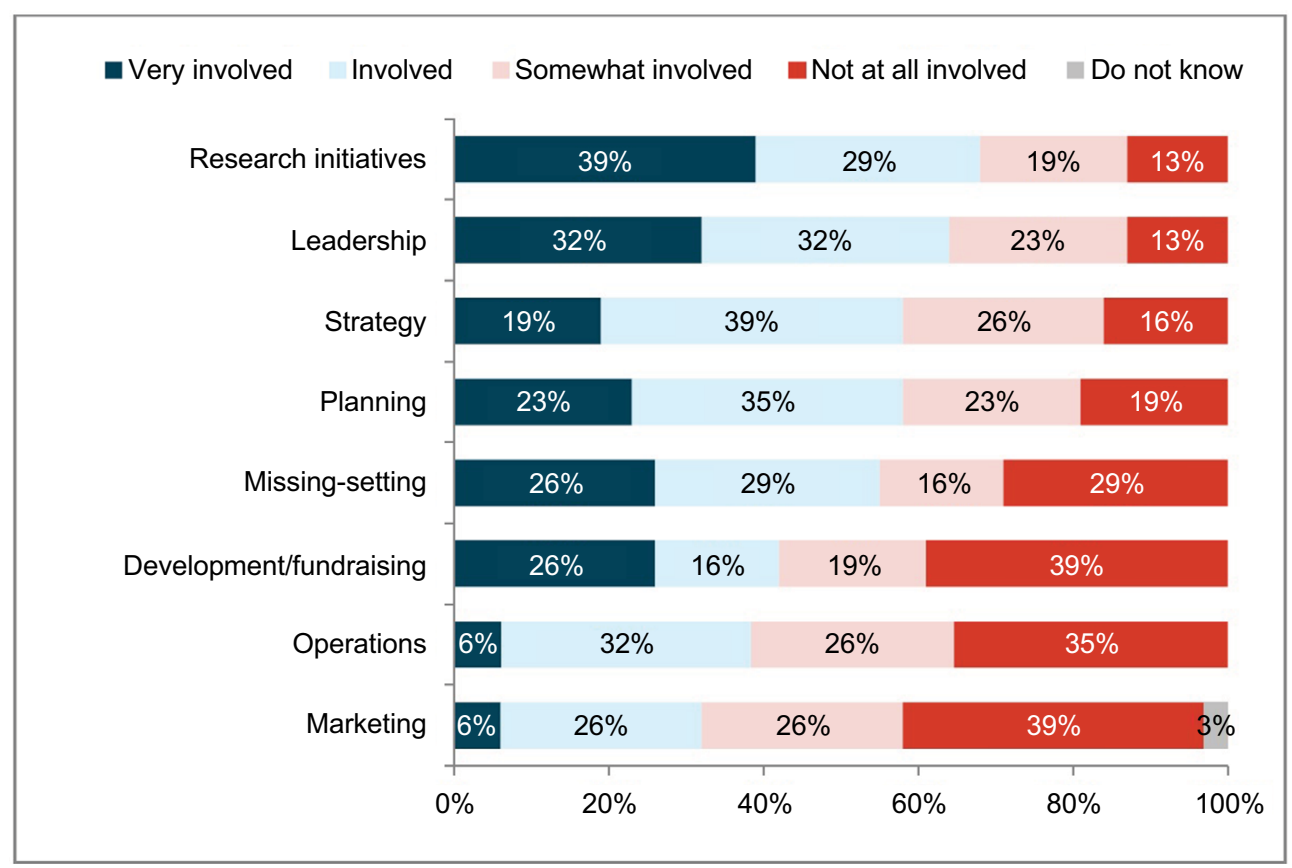

Figure 2 Perceptions of levels of involvement in operational areas.

Note: This chart is adapted from the report of Hanover Research, "Nursing School and AHC Relationship Survey Analysis," commissioned by the University of Washington School of Nursing, March 2015.

\section{Discussion}

\section{The AACN's Manatt Report}

With its emphasis more generally on what needs to change within AHCs themselves to improve their level of care and expanded areas of focus, the AACN survey was different in purpose and goal from the UWSoN survey. The latter is more focused on nursing school leaders' perspectives of their roles within AHCs. Yet, what emerges from a combination of the two is a clear picture of growing trends in how AHCs operate, and how leaders in schools of nursing perceive current conditions and areas for improvement.

The AACN report concluded that AHCs need to:

- Enhance population health competence.

- Further develop and integrate a continuum of care that "links acute, post-acute, and community-based care".

- Develop a foundation of primary care and enhanced patient access.

- Ensure quality, patient safety, and outcomes within the delivery system. ${ }^{5}$

\section{The changing landscape of AHCs and nursing schools' involvement}

Despite the high level of representation by nurses as clinicians on the frontlines of health care delivery, both the UWSoN and AACN survey found that deans and leaders of nursing schools are underrepresented in relation to other partners within AHCs, and they have a secondary or peripheral role in governance and leadership, finance, patient safety, and other key functions of AHCs. Nursing leaders are often not accorded peer status on matters of budget, practice opportunities, status, or patient advocacy. The inordinate focus on graduate medical education with insufficient attention to nursing education and research is one area needing to be addressed if AHCs are to succeed.

Other studies, such as the landmark The future of nursing: leading change, advancing health, have reinforced this perspective. The Robert Wood Johnson Foundation/National Academy of Medicine committee authors stated that achieving health care transformation, " $[\ldots]$ will require remodeling many aspects of the health care system" and explicitly calls for nurses to have a more pronounced role. ${ }^{6}$

Current trends, such as institutional support for population health and implementation of provisions of the Affordable Care Act, exemplify the evolving restructuring of health care systems to adjust to changing federal and state-level mandates, as well as a desire to meet the needs of the populations they serve. In particular, the need to address health inequities and level the playing field as far as socioeconomic determinants of health are signs of the working assumptions inherent in the population health approach.

Both surveys as well as research on interprofessional practice generally find that AHCs will need to become more inclu- 
sive of schools of nursing and other professional organizational partners and become more fully versed in evidence-based treatments if they are to effectively meet the needs of patients and adequately prepare the next generation of students.?

\section{Alignment and realignment}

Health care education is a complex, lengthy, and costly process, as is clinical care. For all these reasons, effecting change in either realm can be difficult.

The optimal outcome results from perfect alignment of all elements in the educational, research, and clinical portions of the system. The first recommendation of the Institute of Medicine's report on AHCs was that AHCs, "take the lead in reforming the content and methods of health professions education to include the integrated development of educational curricula and approaches". ${ }^{8}$

Based on the data derived from the UWSoN survey of ANI deans and the AACN's survey issued by Manatt Health Solutions and other sources, ${ }^{6,9,10}$ and following consultation with colleagues from other schools of nursing, a series of recommendations was generated (Box 1). Leaders of AHCs and of ANIs may wish to reflect upon these recommendations and pursue their objectives in order to achieve greater parity and influence within $\mathrm{ACHs}$, and thereby improve programmatic impact.

\section{Conclusion}

Health care is changing functionally, structurally, and economically. Increasingly, health equity advocates are citing the need for a culture of health. Interpretations of this vary, but at its core is a paradigm shift from an emphasis on treating illness to an emphasis on promoting wellness - an approach that has long characterized nursing. This is a resounding call for fundamental change in American notions of what it means to be healthy and also encompasses an inherent plea for equal access to health care, regardless of sex, gender, economic or other differences in the patient population. ${ }^{8}$

Achieving health care equity must begin with sound structuring of the AHCs, where theory meets practice, and the next generation of policy makers and practitioners is educated and inculcated with the attitudes that are likely to persist throughout their careers.

A relationship of trust and equity between schools of nursing and AHCs is critical to meeting the challenges of health care education, research, and clinical care in the future. The UWSoN survey and the Manatt Report reveal that leaders of most nursing schools currently feel positive about the AHCs with which they are affiliated, but they do not perceive that there is a full partnership or adequate representation when
Box I A platform for integrated AHCs: recommendations for improved alignment

As partners within $\mathrm{AHCs}$, medical centers, biomedical research centers, schools of nursing, and all relevant stakeholders should:

- advocate for, and immediately initiate, discussion of AHC restructuring and culture change, with more equal representation among all participants in health care delivery as the stated goal. Ideally, these discussions would include all health sciences schools (public health, dentistry, pharmacy, etc., as relevant) so that care providers may advocate most effectively on behalf of all patients, as well as within their own specialization.

- Seek ways in which schools of nursing can be fully integrated into all clinical, research, teaching, and financial aspects of the AHC.

- Propose a definite timeline for initiating and completing changes to bring about improved parity between schools of nursing, schools of medicine, and other AHC partners or health sciences schools, in order to enhance patient care and student education which includes attention to population health.

- Commit to being an evidence-based change agent at the school, within the AHC, and in all clinical operations of the AHC.

- Champion programs that place a priority on interprofessional education and interprofessional teams' health care delivery.

- Identify opportunities to derive and generate clinical revenue as part of the AHC health care delivery team.

- Develop research and educational projects which integrate faculty from all professional schools within the AHC.

- Cultivate opportunities for collaboration, consultation, and cooperation between the school of nursing, AHC nursing, and faculty in all other AHC professional schools.

- Seek parity in funding of the health sciences schools from the academic university, as well as from federal grants and institutions which provide research funding opportunities for nursing.

Abbreviation: $\mathrm{AHCs}$, academic health centers.

compared to physicians. In particular, ANI leaders perceive inequity in power or influence compared with schools of medicine.

ANIs are generally accepted as AHC partners in research, but are most often not full partners in governance, strategy creation, practice participation, and financial matters or decision making. While those responding to the survey see nursing as having the second greatest influence after medicine among health care professional schools in providing strategic leadership to AHCs, only 55\% felt that their school of nursing was influential or very influential. Nearly half of all respondents (49\%) expressed dissatisfaction with their nursing school's overall level of involvement with its AHC, and only $13 \%$ indicated they were very satisfied.

With the US health care system in the midst of dramatic change, and connections to population health increasingly being emphasized, AHCs are being called upon to restructure themselves. It is an optimal time for schools of nursing to reassess their role within AHCs and to seek restructuring that makes them full peers and partners in a new AHC model. 
Nursing schools must insist upon greater inclusion, seek allies within schools of medicine and other disciplines, and demonstrate their value. Nursing is a primary rather than ancillary part of the health care solution.

The Institute of Medicine's report report on AHCs said, "Given the magnitude of the changes required by AHCs, it is important that clear goals be set so that progress toward making those changes can be steadily measured". ${ }^{2}$ We concur.

\section{Acknowledgment}

The authors would like to acknowledge the support of Hanover Research for its implementation of the survey and data collection for the University of Washington-commissioned national survey of nursing school deans and nurse leaders in the US, which was the main impetus for this article.

\section{Disclosure}

The authors report no conflicts of interest in this work.

\section{References}

1. American Association of Academic Health Centers [homepage on the Internet]. Criteria for AAHC Membership. AAHC; 2017. Available from: http://www.aahcdc.org/About/Membership. Accessed April 20, 2017.
2. Kohn LT, editor. Academic Health Centers: Leading Change in the 21st Century. Washington: National Academies Press; 2004.

3. World Health Organization [webpage on the Internet]. Primary Health Care: Now More Than Ever. Geneva: World Health Organization; 2008. Available from: http://www.who.int/whr/2008/en/. Accessed April 20, 2017.

4. American Association of Colleges of Nursing. Elevating the Role of Academic Nursing. Conference in Washington, DC; 2015.

5. American Association of Colleges of Nursing [webpage on the Internet]. Advancing Healthcare Transformation: A New Era for Academic Nursing. American Association of Colleges of Nursing; 2016. Available from: http://www.aacn.nche.edu/aacn-manatt-report.pdf. Accessed April 20, 2017.

6. Committee on the Robert Wood Johnson Foundation Initiative on the Future of Nursing at the Institute of Medicine, Robert Wood Johnson Foundation, Institute of Medicine (U.S.). The future of nursing: leading change, advancing health. Washington, DC: National Academies Press; 2011.

7. Lutfiyya MN, Brandt BF, Cerra F. Reflections from the intersection of health professions education and clinical practice: the state of the science of interprofessional education and collaborative practice. Acad Med. 2016;91(6):766-771.

8. Perez B, Szekendi MK, Taylor-Clark K, Vaughn J, Susman K. Advancing a culture of health: population health programs in place at essential hospitals and academic medical centers (2012-2014). J Healthc Qual. 2016;38(2):66-75.

9. Monroe AK, Levine RB, Clark JM, Bickel J, MacDonald SM, Resar L. Through a gender lens: a view of gender and leadership positions in a department of medicine. J Womens Health (Larchmt). 2015;24(10): $837-842$.

10. Wehbe-Janek H, Markova T, Polis RL, Peters M, Liu Y. Preparing academic medical centers for the clinical learning environment review: alliance of independent academic medical centers national initiative IV outcomes and evaluation. Ochsner J. 2016;16(2):166-171.
Journal of Multidisciplinary Healthcare

\section{Publish your work in this journal}

The Journal of Multidisciplinary Healthcare is an international, peerreviewed open-access journal that aims to represent and publish research in healthcare areas delivered by practitioners of different disciplines. This includes studies and reviews conducted by multidisciplinary teams as well as research which evaluates the results or conduct of such teams or health

\section{Dovepress}

care processes in general. The journal covers a very wide range of areas and welcomes submissions from practitioners at all levels, from all over the world. The manuscript management system is completely online and includes a very quick and fair peer-review system. Visit http://www.dovepress.com/ testimonials.php to read real quotes from published authors. 